\title{
Standardized low-resolution electromagnetic tomography of preattentive auditory discrimination in accumulative drinker
}

\author{
Phakkharawat Sittiprapaporn \\ Assistant Professor and Head, Brain Science and Engineering Innovation Research Group, School of Anti-Aging and \\ Regenerative Medicine, Mae Fah Luang University, Bangkok, Thailand, and Department of Anti-Aging Science, School \\ of Anti-Aging and Regenerative Medicine, Mae Fah Luang University, Bangkok, Thailand
}

Background: The present study proposes a task-dependent hypothesis in which the phonological units of segmental (e.g., consonant and vowel) and suprasegmental (e.g., prosodic) are processed in discrete asymmetry of the human brain. Although the left hemisphere (LH) is selectively employed for processing linguistic information irrespectively of acoustic cues or subtype of phonological unit, the right hemisphere $(\mathrm{RH})$ is employed for prosody-specific cues.Although the left hemisphere is selectively employed for processing linguistic information irrespectively of acoustic cues or subtype of phonological unit, the right hemisphere is employed for prosody-specific cues. Aims and Objective: As both hemispheres are lateralized for speech and language, the objective of this study is to extend the investigation of how the pre-attentive processing of vowel duration changes of Thai monosyllabic words in alcoholic drinkers. Materials and Methods: Forty healthy right-handed adults participated in this study. Results: This study found that both rising-to-falling and falling-to-rising tone changes perception elicited MMN between 245-297 msec with reference to the standardstimulus ERPs. The long-to-short and short-to-long vowel duration changes elicited a strong MMN for both groups. Source localization was obtained in the Middle Temporal Gyrus (MTG) of the left hemisphere (LH) for both groups. Conclusion: Automatic detection of changes in contour tones is a useful index of language universal auditory memory traces.

Access this article online

Website:

http://nepjol.info/index.php/AJMS DOI: 10.3126/ajms.v11i2.26580 E-ISSN: 2091-0576

P-ISSN: 2467-9100

Key words: Event-related potential; Sound; Tone; Contour tone; Mismatch negativity

\section{INTRODUCTION}

When engaged in a conversation, listeners tune in to the relevant stream of speech and filter out irrelevant speech input that may be present in the same environment. Nonetheless, attention might be involuntarily diverted to meaningful items coming from an ignored stream, like in the well-known own-name effect. ${ }^{1-2}$ This brings up the question of to what extent speech is processed in the ignored streams. A fundamental, yet controversial issue is whether the human brain contains neural circuits that are uniquely engaged in the early, pre-attentive stage of speech processing. While it seems indisputable that language is sub-served by left-hemisphere (LH) and right-hemisphere
$(\mathrm{RH})$ are lateralized for speech, language, or something else. Hypotheses proposed to account for functional hemispheric asymmetries can generally be classified as either cue dependent i.e., basic neural mechanism underlie processing of complex auditory stimuli regardless of linguistic relevance, ${ }^{3}$ or task dependent, i.e., specialized neural mechanisms exist that are activated only by speech. ${ }^{4}$

The present study proposes a task-dependent hypothesis in which the phonological units of segmental (e.g., consonant and vowel) and suprasegmental (e.g., prosodic) are processed in discrete asymmetry of the human brain. Although the left hemisphere $(\mathrm{LH})$ is selectively employed for processing linguistic information irrespectively of acoustic cues or subtype of phonological unit, the right hemisphere $(\mathrm{RH})$ 
is employed for prosody-specific cues. ${ }^{4}$ The propose of the present study is to use both an auditory MMN component of event-related potential (ERP) recording and the low resolution electromagnetic tomography (LORETA) techniques to measure the degree of cortical activation and to localize the brain area contributing to the scalp recorded auditory MMN component, respectively, during the passive oddball paradigm.

In earlier studies, ${ }^{4-6}$ Chinese and English listeners did not show the same left-hemisphere (LH) lateralization as Thai listeners when making perceptual judgments of Thai tones. In addition, Chinese and English listeners were asked to make perceptual judgments of Chinese tones, consonants, and vowels. Chinese listeners showed left-hemisphere (LH) lateralization for both suprasegmental and segmental phonological units. ${ }^{6}$ These earlier studies suggest that functional circuits engage in early, pre-attentive speech perception of either suprasegmental or segmental units in tone languages. While it seems indisputable that language is sub-served by left-hemisphere ( $\mathrm{LH}$ ) and right-hemisphere $(\mathrm{RH})$ are lateralized for speech, language, or something else. Hypotheses proposed to account for functional hemispheric asymmetries can generally be classified as either cue dependent i.e., basic neural mechanism underlie processing of complex auditory stimuli regardless of linguistic relevance, ${ }^{7}$ or task dependent, i.e., specialized neural mechanisms exist that are activated only by speech. ${ }^{8}$

Previous ERP studies at a phonetic level demonstrated that the MMN was enhanced in Finnish subjects by their first-language (Finnish) phoneme prototype rather than a non-prototype (Estonian) ${ }^{9}$ and that the MMN for a vowel contrast in Finnish was not generated in native Hungarian speakers with no knowledge of Finnish, ${ }^{10}$ implying that the MMN reflects language-specific memory traces formed by early and extensive exposure to a first language. However, language-specific word-related MMN/MMF components at acoustic and phonetic levels remain to be investigated in future studies. The differences between these studies provide the impetus for future investigations of duration processing and temporal integration differences across language groups. Although the left hemisphere is selectively employed for processing linguistic information irrespectively of acoustic cues or subtype of phonological unit, the right hemisphere is employed for prosody-specific cues. ${ }^{8}$ The propose of the present study is, thus, to use both an auditory MMN component of event-related potential (ERP) recording and the standardized Low Resolution Electromagnetic Tomography (sLORETA) techniques to measure the degree of cortical activation and to localize the brain area contributing to the scalp recorded auditory MMN component, respectively, during the passive oddball paradigm. Thus, the objective of this study is to investigate the pre-attentive processing of vowel duration changes in Thai alcoholic drinkers.

\section{MATERIALS AND METHODS}

\section{Participants}

Forty healthy right-handed adults with normal hearing and no known neurological disorders volunteered for participation: twenty non-alcoholic drinkers (Control group), aged 23-39 (mean 27.6 \pm 0.34 ) and twenty alcoholic drinkers (Target group), aged 23-29 (mean 31.18 \pm 1.07 ). The approval of the institutional committee on human research and written consent from each subject were obtained.

\section{Stimuli and procedure}

Stimuli consisted of two pairs of monosyllabic, Thai words. Speech stimuli were digitally generated and edited to have equal peak energy level in decibels SPL with the remaining data within each of the stimuli scaled accordingly using the Cool Edit Pro v. 2.0 (Syntrillium Software Corporation). The sound pressure levels of speech stimuli were then measured at the output of the

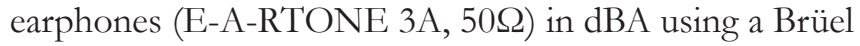
and Kjaer 2230 sound-level meter. Pairs were designed to have similar long vowel duration. Two different stimuli were synthetically generated: Stimuli $1: / \mathrm{k}^{\mathrm{h}} \mathrm{aam} /-$ long vowel; Stimuli 2: $/ \mathrm{k}^{\mathrm{h}} \mathrm{am} /$ - short vowel. Five native speakers listened to the synthesized words and evaluated them all as natural sounding.

The standard (S)/deviant (D) pairs for each trial, which was randomized across participants. The sounds were presented binaurally via headphones (Telephonic TDH-39-P) at $85 \mathrm{~dB}$. The inter-stimulus interval (ISI) was 1.25 second (offset-onset). Deviant stimuli appeared randomly among the standards at $10 \%$ probability. Each experiment included 125 trials $(10 \% \mathrm{D})$. The stimuli were binaurally delivered using SuperLab software (Cedrus Corporation, San Pedro, USA) via headphones (Telephonic TDH-39-P). EEG signal recording was time-locked to the onset of a word. Subjects were instructed not to pay attention to the stimuli presented via headphones, but rather to concentrate on a self-selected silent, subtitled movie.

\section{Electroencephalographic recording}

For EEG/ERP recording, the standard 20 locations of the 10-20 system, EEG, eego ${ }^{\text {TM }}$ (ANT Neuro, Natherlands), was recorded via an electrocap (Electrocap International) from 20 active electrodes (Fp1, Fp2, F7, F3, Fz, F4, T3, C3, Cz, C4, T4, T5, P3, Pz, P4, T6, O1, Oz, O2) positioned according to the 10-20 International System of Electrode Placement, plus $\mathrm{Oz}$ and Ground were applied, 
pre-mounted in an elastic Electro-Cap. Reference electrodes were manually applied to left and right mandibles, where the Fp1 and Fp2 electrodes were used for ocular artifact detection. Horizontal eye movements were monitored with electrodes at the left and right outer canthi and vertical eye movements were monitored at Fp1 and Fp2. EEG was amplified with a gain of 30,000 and filtered with a band pass of 0.1-30 Hz. EEGs were acquired as continuous signals and were subsequently segmented into epochs of 1s (a $100 \mathrm{msec}$ pre-stimulus baseline and a $900 \mathrm{msec}$ poststimulus epoch).

\section{EEG data processing}

The recordings were filtered and carefully inspected for eye movement and muscle artifacts. ERPs were obtained by averaging epoch, which started $100 \mathrm{~ms}$ before the stimulus onset and ended $900 \mathrm{~ms}$ thereafter; the -100-0 ms interval was used as a baseline. Epochs with voltage variation exceeding $\pm 100 \mu \mathrm{V}$ at any EEG channel were rejected from further analysis. The MMN was obtained by subtracting the response to the standard from that to the deviant stimulus. All responses were recalculated offline against average reference for further analysis.

\section{LORETA and dipole computations}

Low-resolution Electromagnetic Tomography (LORETA) is the new implementation of LORETA in the Talairach brain. LORETA makes use of the three-shell spherical head model registered to the Talairach human brain atlas, ${ }^{11}$ available as a digitized MRI from the Brain Imaging Center, Montreal Neurologic Institute. Registration between spherical and realistic head geometry use EEG electrode coordinates reported by Towle et al. ${ }^{12}$ The solution space is restricted to cortical gray matter and hippocampus, as determined by the corresponding digitized Probability Atlas also available from Brain Imaging Center, Montreal Neurologic Institute. A voxel is labeled as gray matter if it meets the following three conditions: its probability of being gray matter is higher than that of being white matter, its probability of being gray matter is higher than that of being cerebrospinal fluid, and its probability of being gray matter is higher than $33 \%$. Only gray matter voxels at $7-\mathrm{mm}$ spatial resolution are produced under these neuroanatomical constraints. LORETA computations use the exact head model determined from each individual subject's MRI. The final step in any analysis procedure would be to cross-register the individual's anatomical and functional image to the standard Talairach atlas.

\section{Brodmann area (BA) and brain regions localization procedure}

Regarding to the Brodmann areas(s) and brain regions localization, the Talairach Daemon (TD) will be taken into consideration. The Talairach Daemon (TD) is a high-speed database server for querying and retrieving data about human brain structure over the Internet (http:/ / ric.uthscsa. edu/td_applet/). The TD server data is searched using $x-y-z$ coordinates resolved to $1 \mathrm{x} 1 \mathrm{x} 1 \mathrm{~mm}$ volume elements within a standardized stereotaxic space. An array, indexed by $x-y-z$ coordinates, that spans $170 \mathrm{~mm}(\mathrm{x}), 210 \mathrm{~mm}$ (y) and $200 \mathrm{~mm}(\mathrm{z})$, provides high-speed access to data. Array dimensions were selected to be approximately 25\% larger than those of the Co-planar Stereotaxic Atlas of the Human Brain. ${ }^{11}$ Coordinates tracked by the TD server are spatially consistent with the Talairach Atlas. Each array location stores a pointer to a relation record that holds data describing what is present at the corresponding coordinate. Presently, the data in relation records are either Structure Probability Maps (SP Maps) or Talairach Atlas Labels, though others can be easily added. The relation records are implemented as linked lists to names and values for brain structures. The TD server is run on a Sun SPARCstation 20 with 200 Mbytes of memory. Intention is to provide 24hour access to the data using a variety of client applications, as well as continue to add more brain structure information to the database.

\section{Spatial analysis}

The average MMN latency was defined as a moment of the global field power with an epoch of 50-ms time window related stable scalp-potential topography. ${ }^{13}$ In the next step, standardized Low-resolution Electromagnetic Tomography (sLORETA) was applied to estimate the current source density distribution in the brain, which contributed to the electrical scalp field. ${ }^{13}$ Maps are computed with sLORETA. sLORETA computed the smoothest of all possible source configurations throughout the brain volume by minimizing the total squared Laplacian of source strengths. ${ }^{13}$

\section{Data analysis}

During the auditory stimulation, electric activity of the subjects' brain was continuously recorded. The MMN was obtained by subtracting the response to the standard (rising-to-falling OR falling-to-rising) from that to the deviant (rising-to-falling OR falling-to-rising) stimulus. The statistical significance of MMN was tested with one sample t-test.

\section{RESULTS}

The grand-averaged ERPs show that both long-to-short and short-to-long vowel duration changes perception elicited MMN between 245-297 ms with reference to the standard-stimulus ERPs. The t-test comparing MMN amplitudes of the standard and deviant were statistically significant (e.g., control group ( $-0.57 \pm 0.17)$, target group $(-0.91 \pm 0.43) ; \mathrm{t}(39)=2.16, \mathrm{p}<0.05$ in long-to-short vowel 
changes, and Control group (-0.53 \pm 0.26$)$, target group $(-0.75 \pm 0.39) ; \mathrm{t}(39)=2.58, \mathrm{p}<0.05$ in short-to-long vowel changes) (Table 1 and Figure 1). The result showed that long-to-short and short-to-long vowel duration changes elicited a strong MMN for both control and target groups (Figures 2 and 3).

Source localization analyses were performed using sLORETA. ${ }^{8}$ Table 2 demonstrates the xyz-values in Talairach space as calculated with sLORETA in the time window 245-297 msec. In long-to-short vowel duration changes, a single source was estimated to be located in the Middle Temporal Gyrus (MTG) of left hemisphere (LH) for both groups. In short-to-long vowel duration changes, a single source was obtained in the Middle Temporal Gyrus (MTG) of left hemisphere (LH) for both groups (Table 2 and Figures 2 and 3).

\section{DISCUSSION}

Both long-to-short and short-to-long vowel duration changes perception elicited MMN between 245-297 ms with reference to the standard-stimulus ERPs. The long-to-short and short-to-long vowel duration changes elicited a strong MMN for control and target groups. Source localization

\begin{tabular}{lcc} 
Table 1: Mean amplitude $(\mu \mathrm{V}) \pm \mathrm{S}$.D. of MMN \\
elicited by a vowel duration changes with level \\
tone perception in control and target groups \\
\hline Vowel duration & \multicolumn{2}{c}{ Participants } \\
\cline { 2 - 3 } & Control group & Target group \\
\hline Long-to-short vowel & $-0.57 \pm 0.17$ & $-0.91 \pm 0.43$ \\
Short-to-long vowel & $-0.53 \pm 0.26$ & $-0.75 \pm 0.39$ \\
\hline
\end{tabular}

analyses performed using sLORETA demonstrates that sources were obtained in the Middle Temporal Gyrus (MTG) of the left hemisphere (LH) for both groups. The main finding of the present study also indicates that the prominent response to across- and within-category change of vowel in consonant-vowel syllable elicited MMN peaking at 245-297 ms from stimulus onset. The magnitude of the acoustic difference between the stimulus pairs was reflected by the MMN amplitude. The MMN amplitude was larger in across- than within-category vowel in consonantvowel syllable contrasts. This can be the evidence for the left-hemispheric phoneme representation in the human auditory cortex. The converging evidence from factorial statistics of hemispheric effects on sLORETA values around the MMN peaks provide further support for the significance of these pre-attentively activated hemispheric differences in vowel category discrimination.

For a long time, the EEG inverse problem could not find a satisfactory solution. As verified by point spread function simulations, sources could be reconstructed at best with some non-systematic localization error. sLORETA was found to have zero localization error, ${ }^{14}$ hence it finally solved exactly the EEG inverse solution problem (as far as localization error in the absence of noise is concerned). The present study thus used standardized LORETA space consisting in 2394 voxels of size $7 \times 7 \times 7 \mathrm{~mm}$. The spatial dispersion of the reconstruction with 21 electrodes is considerable with the zero localization. With respect to the usefulness of a new tomographic source analysis, the present data showed that the sLORETA can locate multiple non-dipolar sources accurately to demonstrate the dynamic, alternating between left and right homologous regions for different category of vowels in language processing.

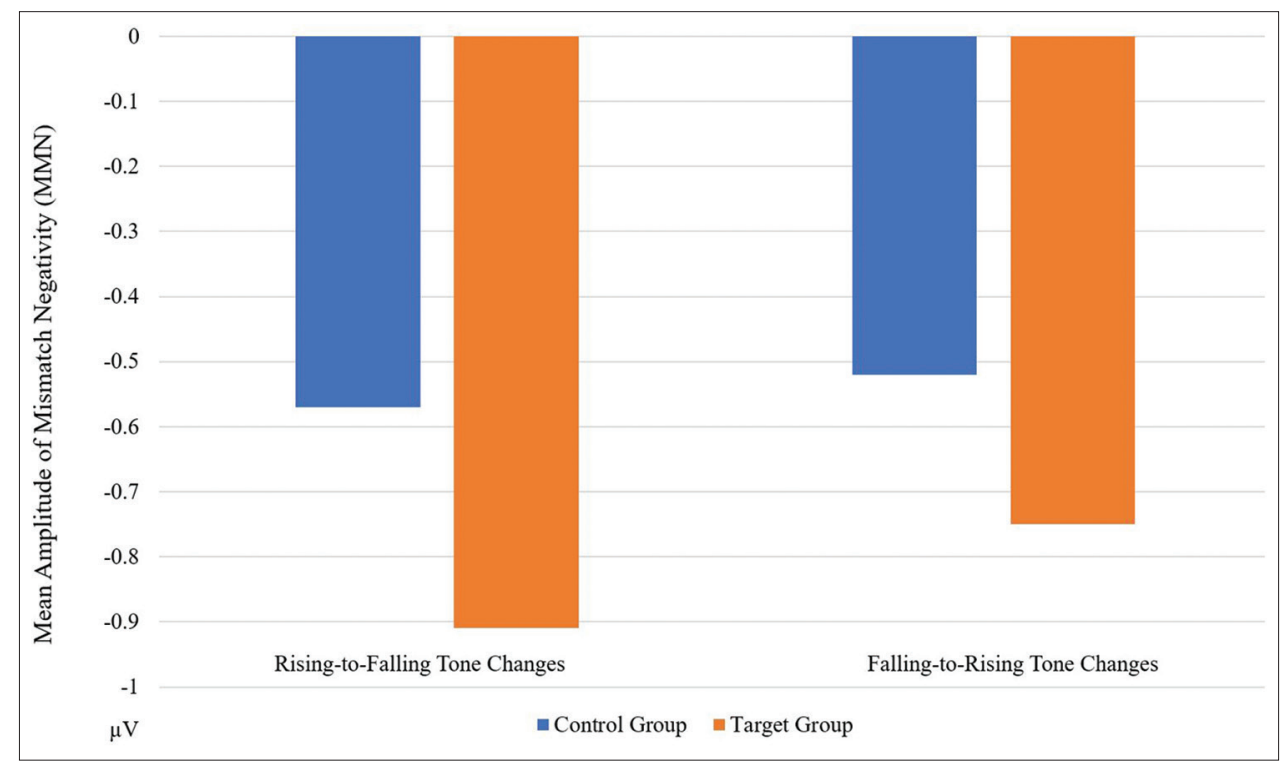

Figure 1: Mean amplitude $(\mu \mathrm{V}) \pm$ S.D. of MMN elicited by a vowel duration changes with level tone perceptionin control and target groups 
This was followed by activation of the homologous right hemisphere region for within-category vowel change. ${ }^{15-17}$ The different pattern of activation to within-category and across-category changes of vowels support the view that information transfer in the right and left hemispheres are for analysis of different aspects: physical features within the same phoneme boundary in the right and independent phonemes in the left hemisphere.

The present results support a model suggesting that in the intact brain, each hemisphere relies on earlier processing stages in both hemispheres and on the ongoing evolving stimulus, which contribute to searching the lexicon. This model is compatible with separate right and left hemispheric distributions of neural networks, interconnected and using

\begin{tabular}{|c|c|c|c|c|c|}
\hline \multirow{2}{*}{$\begin{array}{l}\text { Vowel duration } \\
\text { changes }\end{array}$} & \multicolumn{3}{|c|}{ Coordinates (mm) } & \multirow[t]{2}{*}{ BA } & \multirow[t]{2}{*}{$t$ values } \\
\hline & $x$ & $y$ & $z$ & & \\
\hline \multicolumn{6}{|c|}{ Long-to-short vowel } \\
\hline Control group & -52 & -67 & 8 & 39 & 2.23 \\
\hline Target group & -52 & -67 & 8 & 39 & 1.26 \\
\hline \multicolumn{6}{|c|}{ Short-to-long vowel } \\
\hline Control group & -52 & -67 & 8 & 39 & 2.52 \\
\hline Target group & -59 & -32 & 15 & 22 & 1.23 \\
\hline
\end{tabular}

the processing of the other hemisphere's output as input for its computations. The time scale of inter-hemispheric transfers suggests that information is transferred back and forth between left and right homologous regions. The extensive information exchanges between right and left homologous sites suggest that as a word is evolving over time, right and left hemisphere regions contribute and interact to create efficient linguistic parallel processing. ${ }^{15}$ This may include the transfer of information to the right hemisphere to free resources in the left one to focus on the meanings ${ }^{15-16}$ or to process further input as the word's sounds progress over time. ${ }^{17}$ Thus, the bilateral activation in STG of different category vowel change perception was compatible with previous study ${ }^{18}$ that two possible time courses may be involved: (1) both hemispheres are active in parallel with the left hemisphere dominant for different phonemes boundaries and (2) hemispheric dominance may alternate to play a specific role at different stages of linguistic processing. This supports the hypothesis that the part of superior temporal gyrus contains neural networks specialized for language processing. ${ }^{18}$

The same results were obtained in MEG studies using both tone and Japanese words. ${ }^{19,20}$ The present results parallel the finding in previous studies. ${ }^{19,20}$ The present study, the detection of tone changes was most likely acoustically driven rather than semantically driven, such

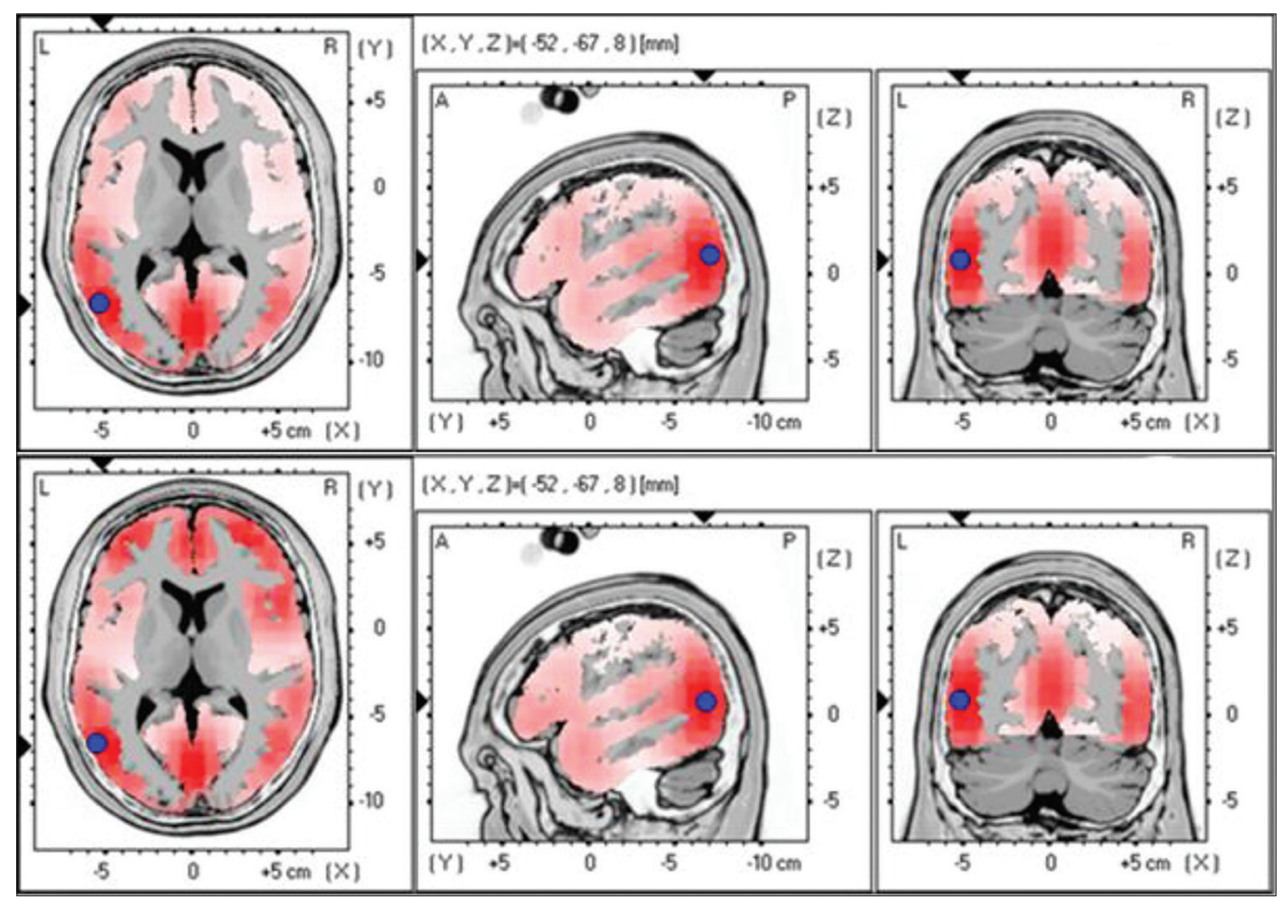

Figure 2: Graphical representation of the standardized Low-resolution Electromagnetic Tomography (sLORETA t-statistic comparing the event-related potentials for mismatch negativity (MMN) responses at the time point of the individual peak over Fz for the long-to-short duration changes of vowels with level tone of control group (top) and target group (bottom) activated in left hemisphere (LH). Red color indicates local maxima of increased electrical activity in an axial, a saggital and a coronal slice through the reference brain. Blue dots mark the center of significantly increased electric activity 


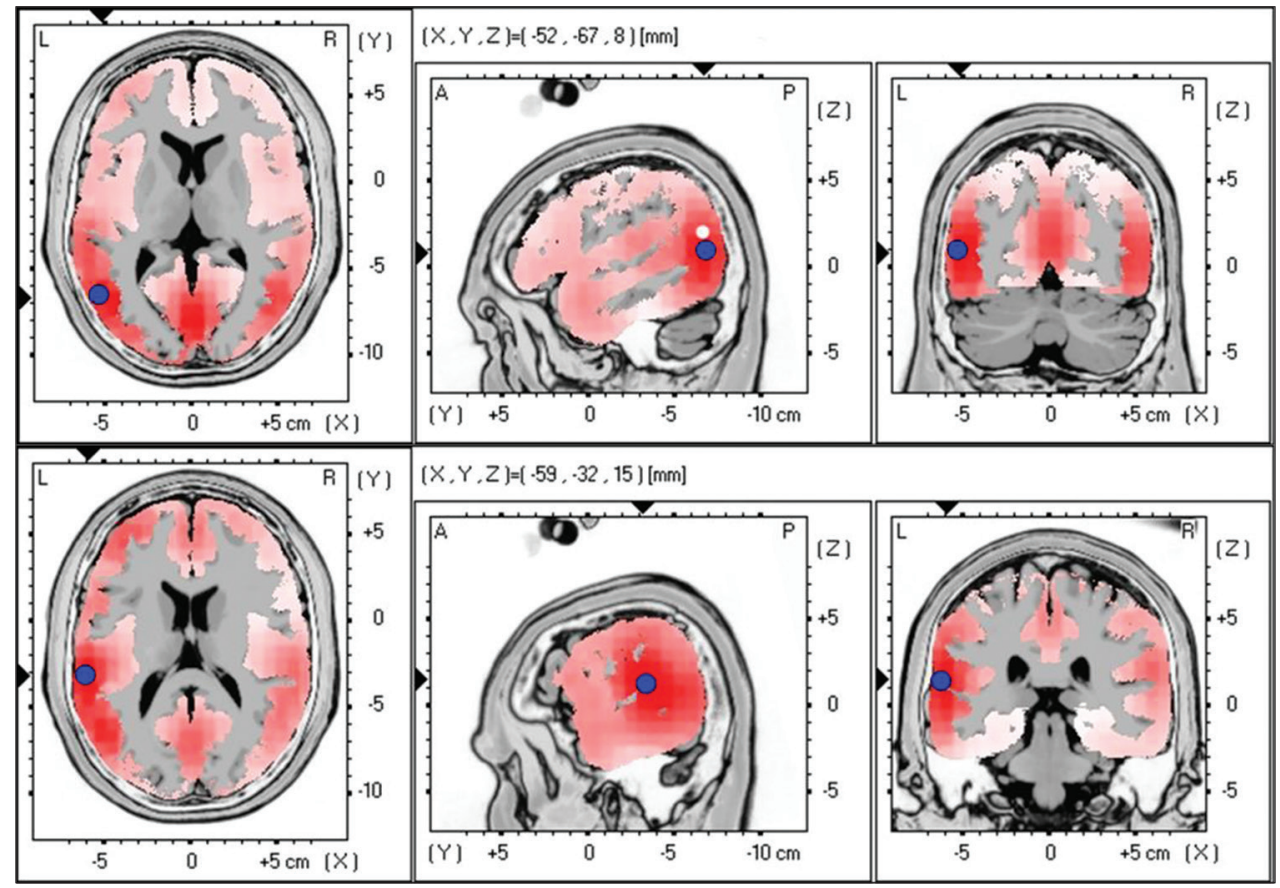

Figure 3: Graphical representation of the standardize Low-resolution Electromagnetic Tomography (sLORETA t-statistic comparing the eventrelated potentials for mismatch negativity (MMN) responses at the time point of the individual peak over Fz for the short-to-long duration changes of vowels with level tone of control group (top) and target group (bottom) activated in left hemisphere (LH).Red color indicates local maxima of increased electrical activity in an axial, a saggital and a coronal slice through the reference brain. Blue dots mark the center of significantly increased electric activity

that the stimuli were processed without any access to semantic information. The acoustic aspect in the absence of phonetic or higher-order properties may account for why target group had similar neuronal responses to control group. This suggests that at the point of stimulus disparity or thereafter, change detection of tone changes is somehow compromised. It is reasonable to speculate that the continued auditory processing required for the tone changes interferes with or masks the detection mechanism underlying the $\mathrm{MMN} .{ }^{19}$ In the same way, the current findings showed similar results with previous tone studies that reported a clear MMN elicited by both duration increments and decrements ${ }^{21}$ and a larger MMN elicited by increments than decrements. ${ }^{22}$ The MMN component in the present study was also found to be more sensitive to control group compared to target group.

One might expect language-specific effects on the elicitation of the MMN in speech, since Thai is a tonal language and English is a stress-accent language. A tendency towards stronger MMN in target group is observed and support such an expectation in that statistically significant difference in MMN amplitudes was found between groups at an acoustic level. Previous ERP studies at a phonetic level demonstrated that the MMN was enhanced in Finnish subjects by their first-language (Finnish) phoneme prototype rather than a non-prototype (Estonian) ${ }^{5}$ and that the MMN for a vowel contrast in Finnish was not generated in native Hungarian speakers with no knowledge of Finnish, ${ }^{10}$ implying that the MMN reflects language-specific memory traces formed by early and extensive exposure to a first language. However, language-specific word-related MMN/MMF components at acoustic and phonetic levels in both groups remain to be investigated in future studies.

\section{CONCLUSION}

Both rising-to-falling and falling-to-rising tone changes perception elicited MMN between 217-264 ms with reference to the standard-stimulus ERPs. The risingto-falling and falling-to-rising tone changes elicited a strong MMN bilaterally for control and target groups. Source localization analyses performed using sLORETA demonstrates that sources were obtained in the Middle Temporal Gyrus (MTG) of the right hemisphere (RH) for both groups. Automatic detection of changes in tone changes is a useful index of language universal auditory memory traces.

\section{ACKNOWLEDGEMENT}

This research was supported by research funding from Mae Fah Luang University grant (MFU-grant no. 61210320031), Mae Fah Luang University grant (Electroencephalogram Laboratory 2019), and Brain Science and Engineering 
Innovation Research Group, Mae Fah Luang University grant (MFU-grant no. 611U109005), Thailand.

We thank all of the subjects involved in this research as main data provider of this studies.

\section{REFERENCES}

1. Monrad-Krohn GH. Dysprosody or altered melody of language. Brain 1947; 70: 405-423.

2. Monrad-Krohn GH. The third element of speech: Prosody and its disorders. In Halpern, L. (Ed.), Problems in Dynamic Neurology, pp 101-118. Hebrew University Press: Jerusalem; 1963.

3. Levy J. Psychobiological implications of bilateral asymmetry. In: Dimond SJ. Beaumont JG. Editors. Hemisphere function in the human brain. New York: Wiley; 1974.

4. Hsieh L, Gandour J, Wong D and Hutchins GD. Functional Heterogeneity of inferior Gyrus is Shaped by Linguistic Experience. Brain and Language 2001; 10: 1-15.

5. Gandour J. Aphasia in tone languages. In Coppens P, Basso A, Lebrun Y (Eds.), Aphasia in atypical populations (pp 117/141). Hillsdale, NJ: Lawrence Erlbaum, 1998.

6. Gandour J, Wong D and Hsieh L. A Cross-linguistic PET Study of Tone Perception. Journal of Cognitive Neuroscience 2000; 12(1): 207-222.

7. Ivry $\mathrm{R}$ and Roberson $\mathrm{L}$. The two sides of perception. Cambridge, MA: MIT Press; 1998.

8. Imaizumi S Mori K, Kiritani S, Hosoi H and Tonoike M. Taskdependent laterality for cue decoding during spoken language processing. Neuro Report 1998; 9: 899-903.

9. Näätänen R. Attention and Brain Function. Lawrence Erlbaurn, Hillsdale, 1992.

10. Winkler I, Lehtokoski A, Alko P, Vainio M, Czigler I, Csepe V, et al. Pre-attentive detection of vowel contrasts utilizes both phonetic and auditory memory representations. Cognitive Brain Research 1999; 7: 357-369.
11. Talairach $\mathrm{J}$ and Tournoux P. Co-planar stereotaxic atlas of the human brain, Stuttgart? Thieme; 1988

12. Towle VL, Bolanos J, Suarez D, Tan K, Grzesczuk R, Levin DN, et al. The spatial location of EEG electrodes: locating the bestfitting sphere relative to cortical anatomy. Electroenceph Clin Neurophysiol 1993: 86: 861-866.

13. Pascual RD, Michel $\mathrm{CM}$ and Lehmann D. Low Resolution Electromagnetic Tomography: A New Method for Localizing Electrical Activity in the Brain. International Journal of Psychophysiolog 1994; 18: 49-65.

14. Pascual RD, Michel $\mathrm{CM}$ and Lehmann D. Low Resolution Electromagnetic Tomography: A New Method for Localizing Electrical Activity in the Brain. International Journal of Psychophysiology 1994; 18: 49-65.

15. Sinai $A$ and Pratt $H$. High-resolution time course of hemispheric dominance revealedby low-resolution electromagnetic tomography. Clinical Neurophysiology 2003; 114:1181-1188.

16. Coney J and Evans KD. Hemispheric asymmetries in the resolution of lexical ambiguity. Neuropsychology 2000; 38:272-282.

17. Knosche TR, Maess $B$ and Friederici AD. Processing of syntactic information monitored by brain surface current density mapping based on MEG. Brain Topogr 1999; 12(2):75-87.

18. Jäncke L, Wustenberg $\mathrm{T}$, Scheich $\mathrm{H}$ and Heinze H-J. Phonetic perception and thetemporal cortex. Neuroimage 2002; 15:733-746.

19. Inouchi M, Kubota M, Ferrari $P$ and Roberts $T$. Neuromagnetic auditory cortex responses to duration and pitch changes in tones: cross-linguistic comparisons of human subjects in directions of acoustic changes. Neuroscience Letter 2002; 331: 138-142.

20. Inouchi M, Kubota M, Ferrari $\mathrm{P}$ and Roberts T. Magnetic mismatch fields elicited by vowel duration and pitch changes in Japanese words in humans: comparison between native- and non-speakers of Japanese. Neuroscience Letter 2003; 353: 165-168.

21. Näätänen $R$, Paavilainen $P$ and Reinikainen $K$. Do event-related potentials to infrequent decrements in duration of auditory stimuli demonstrate a memory trace in man? Neuroscience Letter 1989; 107: 347-352.

22. Jaramillo M, Alku P and Paavilainen P. An event-related potential (ERP) study of duration changes in speech and non-speech sounds. Neuroreport 1990; 10: 3301-3305.

\section{Author's Contribution:}

PS-Concept and design of the study, statistically analyzed and interpreted, manuscript preparation, critical revision of the manuscript.

Work attributed to: Brain Science and Engineering Innovation Research Group School of Anti-Aging and Regenerative Medicine, Mae Fah Luang University, Thailand.

Orcid ID:

Dr. Phakkharawat Sittiprapaporn - (i) https://orcid.org/0000-0002-4103-9396

Source of Support: Mae Fah Luang University grant (MFU-grant no. 61210320031); Mae Fah Luang University grant (Electroencephalogram Laboratory 2019); Brain Science and Engineering Innovation Research Group, Mae Fah Luang University grant (MFU-grant no. 611U109005), Thailand, Conflict of Interest: None. 